\title{
Habilidad escalar del espacio, absorción como medida de centralidad
}

\author{
Spatial scaled ability, absorption as a measure of centrality
}

<RESUMEN>

Este artículo explora hasta que punto es preciso describir los centros suburbanos de Londres como ambientes multiescalares, y que significa esto en términos del tipo de entidad social que es un suburbio. La investigación estudia el fenómeno espacial de «centralidades escalares» dentro de entidades mayos al interior de una ciudad, a través del estudio de veinte centros suburbanos en la ciudad de Londres.

El artículo explora la relación entre la morfología espacial del centro suburbano y su funcionamiento socioeconómico. Por un lado los centros han crecido y se han encogido en el tiempo, pero también han cambiado sus actividades y se han diversificado. Por otro lado Londres ha sido descrita muchas veces como una ciudad de poblados absorbidos, y el estudio propone que existe un ugrado de absorción» el cual tiene que ver con esas diferencias y jerarquías. Se propone una concepción escalar de la centralidad estableciendo conexiones entre patrones morfológicos escalares y funcionamiento socioeconómico. ¿Existe algún patrón escalar, o grado de absorción, en la definición de un centro suburbano? El estudio responde algunas de estas preguntas usando varias de las herramientas desarrolladas por Space Syntax.

$<A B S T R A C T>$

This article explores to what extent it is accurate to describe Greater London's suburban town centres as multi-scaled environments, and what this means in terms of the kind of social entity a suburb is. The research addresses the understanding of the socio-spatial phenomenon of uscaled centralities» within larger entities of a city through the study of twenty suburban town centres in the city of London.

The relationship between suburban town centre spatial morphology and their socio-economic functioning is explored. On the one hand town centres have grown and reduced in size over time, but they also have changed their activities and diversify. On the other hand, London has been described as a city of absorbed villages, and the study proposes that the degree of absorption has to do with these differences and hierarchies. A scaled notion of centrality is explored establishing linkages between morphological scaled patterns and socio-economic functioning. Is there any scale pattern, or absorption degree, in suburban town definition? This study answers some of these questions using various of the space syntax tools.

<PALABRAS CLAVE>

DISEÑO BASADO EN EVIDENCIA / SUBCENTRALIDAD ABSORCIÓN / ESCALAS DE MOVIMIENTO / SINERGIA

KKEY WORDS >

EVIDENCE-BASED APPROACH TO DESIGN / SUB-

CENTRALITY / ABSORPTION / SCALES OF MOVEMENT / SYNERGY
Este artículo expone las principales ideas de la tesis presentada para obtener el título de Master in Science in Advanced Architectural Studies en University College of London (UCL), septiembre 2007. La investigación se llevó a cabo en el marco del financiamiento EPSRC (Engineering and Physical Sciences Research Council) para el proyecto SSTC (Successful Suburban Town Centre) a cargo de Dr. Laura Vaghan en UCL. La investigación explora la relación entre la espacialidad de la forma suburbana y su habilidad para sustentar su funcionamiento como subcentros.

En el estado actual de las ciudades el concepto de suburbia necesita ser entendido desde la multiplicidad de escalas desde las cuales las ciudades son abordables. La condición de subcentro de un lugar no es un fenómeno estático sino que requiere de una perspectiva

\footnotetext{
Arquitecto Universidad de Chile 2001. Recibe la distinción President Medal Award RIBA 2003 a Proyectos de Título. Entre el año 2002 y el 2006 se desempeñó profesionalmente en la oficina Sabbagh Arquitectos. Revista CA y Revista De Arquitectura de la Universidad de Chile, además de desarrollar tareas de docencia en la Facultad de Arquitectura y Urbanismo de la Universidad de Chile entre el año 2000 y el año 2008.
} 


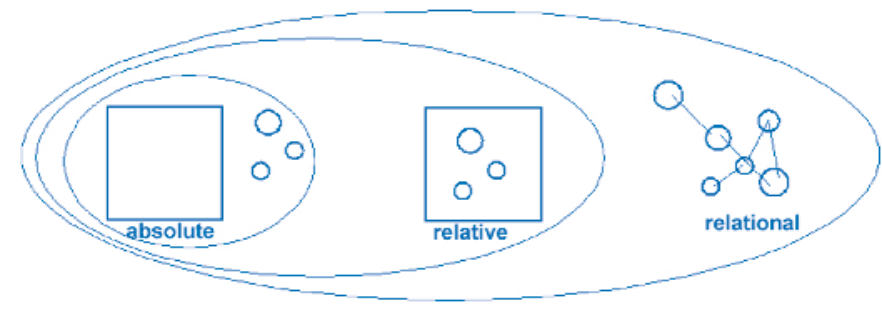

Diagrama 1: Visión Relacional del espacio conteniendo Io Relativo y lo Absoluto.

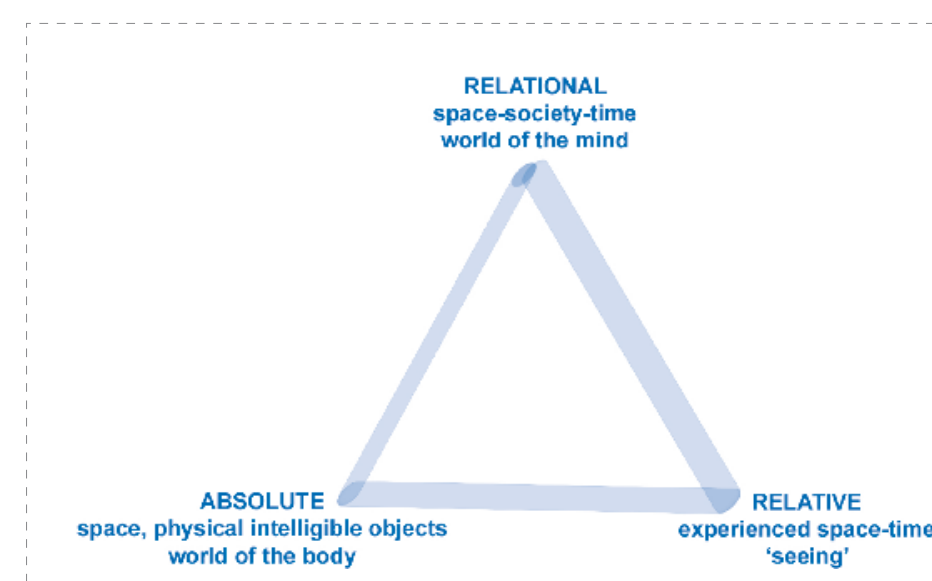

Diagrama 2: Relación entre las tres visiones del espacio. multiescalar para su comprensión. El estudio propone que las cualidades y jerarquías de los subcentros urbanos pueden ser explicadas como función del grado de absorción de cada caso con respecto del total del sistema espacial en el que están insertos. Este fenómeno puede ser medido a través de un índice de absorción, el cual refleja el potencial de un lugar para ser usado en los movimientos locales y globales de los habitantes de una ciudad. En este sentido la centralidad es un proceso, no un estado, en el que las distintas etapas dependen de condiciones configuracionales y morfológicas.

El estudio se sustenta en el desarrollo de las teorías y softwares desarrollados por Space Syntax (SS) ${ }^{1}$ en UCL. Éstas proponen que las particularidades morfológicas y configuracionales del espacio conllevan relaciones estadísticas con las diferentes escalas de movimiento que ocurren en la ciudad e influencian probabilísticamente la capacidad de estos flujos para divergir o superponerse (habilidad escalar). Basándose en estas exploraciones previas, la tesis propone una relación entre patrones espaciales escalares (discursivos a través de dichos softwares) y el carácter y cualidad de los suburbios, expresadas en este estudio a través de su actividad socioeconómica. La calidad y cantidad de actividad suburbana se entiende como señalización de construcción de lugar.
SS propone una relación bidireccional entre el espacio y la sociedad; existe una lógica espacial inherente a nuestras formas sociales, y a su vez el espacio encubre una genética social a través de su configuración. Si el espacio es social en su definición, y la sociedad es intrínsicamente espacial, en la medida que esta simbiosis se mantenga en sombra las propuestas y proyectos que desarrollamos carecerán de efectividad y no lograrán el impacto que buscamos e imaginamos. El espacio entonces no es entendido como un producto social a la manera de Lefebvre ${ }^{2}$ quien examina las sociedades a través de los espacios que éstas han producido basado en sus modos de producción, ni tampoco como lo concibe Giddens $^{3}$ quien otorga al espacio un rol secundario entendiéndolo como un proyección externa. Históricamente la relación espaciosociedad ha sido entendida observando a la sociedad e intentando revelar el espacio desde sus productos, SS al contrario propone esta relación space-first; el espacio desde este punto de vista es generador y activador de procesos sociales.

El espacio, además de estar definido por invariantes como el espacio absoluto de Newton, encuentra estructura en la presencia relativa de objetos y eventos distribuidos en él. Este no sólo tiene injerencia sobre la materia en el sentido entendido por la teoría de la relatividad, sino que las relaciones en y del espacio finalmente son la forma y función de nuestras ciudades. Esto implica definir el espacio relacionalmente, incluyendo el espacio absoluto Newtoniano y el relativo de Leibniz. La definición relacional del espacio propone que el espacio está contenido en la materia y que un objeto representa en si mismo relaciones con otros objetos y sus variaciones a través del tiempo ${ }^{4}$. Como el espacio se manifiesta a través de objetos, es en las relaciones entre ellos que encuentra materialización.

Estas teorías encuentran sustento en una serie de softwares que incrementan el nivel tecnológico disponible, con el objetivo de elevar las complejas relaciones espaciales a un nivel discursivo en el que podamos analizar de manera explícita las potencialidades de nuestras intervenciones ${ }^{5}$. En este sentido es que las herramientas que ofrecen modifican el proceso de diseño, transformando nuestras decisiones intuitivas en argumentos debatibles a nivel de discurso consciente. Depthmap ${ }^{6}$, Webmap ${ }^{7}$ y Confeego ${ }^{8}$ son algunos de los softwares principales que grosso modo, estudian el espacio como redes de relaciones. Finalmente asignan cualidades a los espacios no en base a sus propiedades intrínsecas sino por la manera en que se relacionan con el sistema total. Estas herramientas analizan tanto

SS es un colectivo de teorías y herramientas sobre el espacio desarrolladas en UCL durante los '80 (Hillier \& Hanson, 1984).

Lefebvre, H. The production of space. Londres: Blackwell, 1974.

Giddens, A. The constitution of society. Cambridge, Inglaterra: Polity, 1986.

Harvey, D. Space as a key word. Londres: Paper for Marx and Philosophy, 2004.

Hillier, B. Space is the machine, a configurational theory of architecture. Nueva York: CUP, 1996

Turner, A. Visibility graph analysis. Londres (http://www.vr.cl.ac.uk/research/vga/Vaughan.2004).

Dalton, N. Webmap. Londres: Ovinity Ltd, 2002.

8 Turner A. New developments in Space Syntax software. Istanbul: Faculty of Architecture, 2007. 


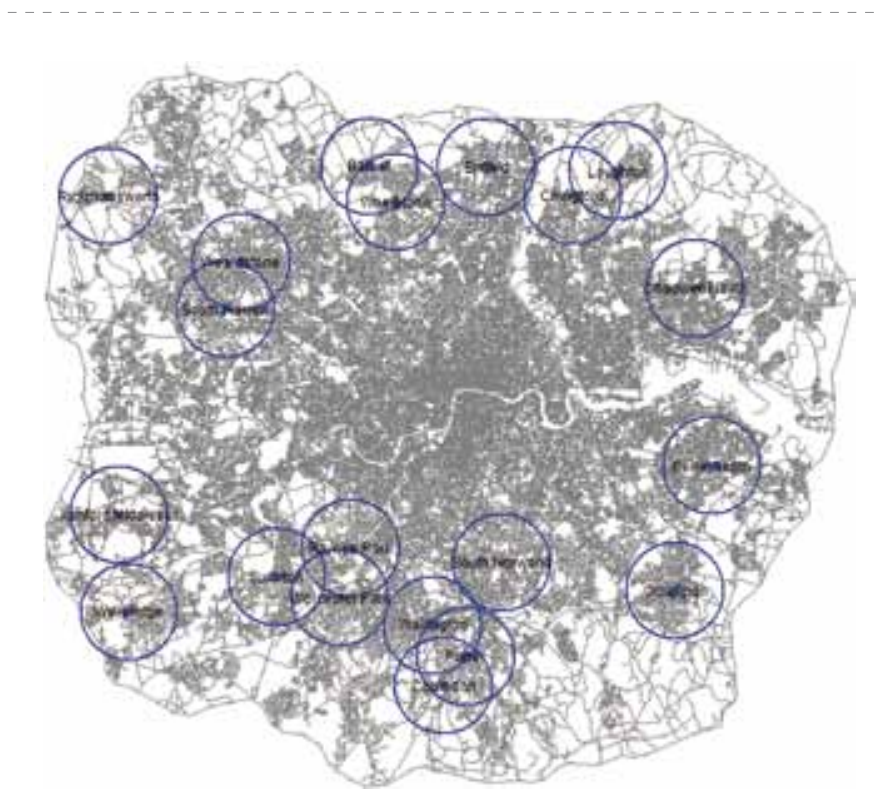

Figura 1: Veinte casos estudiados.

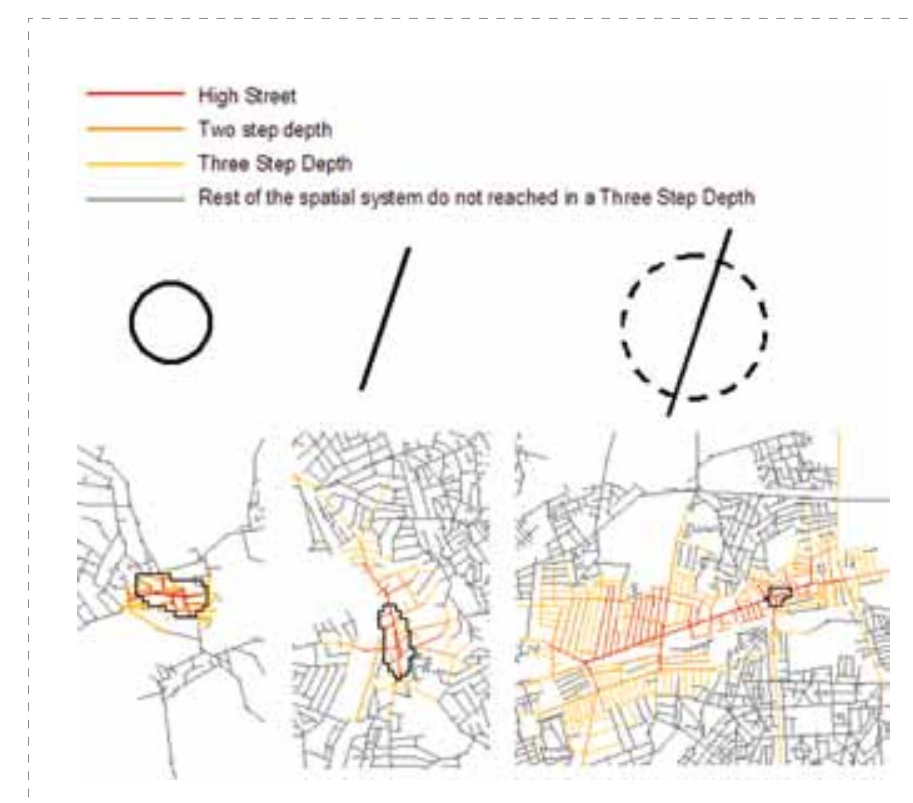

Figura 2: Step depth. el medioambiente construido como futuras propuestas basadas en una aproximación space-first y evidence-based sobre el proceso de diseño.

Este artículo presenta algunas de las ideas producto del estudio de veinte centros suburbanos en la periferia de Londres, como una exploración del potencial de las herramientas desarrolladas por SS para transformar ciertas intuiciones no-discursivas en argumentos y discursos alimentadores del proceso de diseño. ¿Existen reglas específicas subyacentes a la creación de suburbia en la ciudad? ¿Hay alguna lógica tras su configuración espacial? ¿Cuál es la tensión entre urbanidad y suburbanidad? ¿Cómo las estructuras espaciales de los centros suburbanos responden a sus requerimientos funcionales y sociales? De manera más específica el estudio utilizó estas herramientas para develar patrones entre las distintas escalas de movimiento que cruzan el sistema urbano y la morfología de los centros. Si no hay sociedad a-espacial y no hay espacio a-social es precisamente en esa relación que la arquitectura y la ciudad se fundan.
El estudio entiende la subcentralidad como lugares multiescalares poniendo a prueba tres ideas principales: primero, que la subcentralidad no es una condición estática sino un proceso con fases dinámicas que dependen del grado de absorción de cada caso con respecto al total del sistema espacial; segundo, que la jerarquía de los elementos suburbanos (mercado, municipalidad, high stree ${ }^{\ominus}$, comercio, iglesias, industria, bibliotecas y estación de transporte) está relacionada a las etapas y estados del proceso, y tercero; que la localización y distribución de la actividad suburbana también está relacionada al proceso escalar de centralidad suburbana. Las formas tanto urbanas como suburbanas son parte de las probables configuraciones espaciales que las sociedades han desarrollado para dar forma física a sus requerimientos sociales. En este sentido, suburbia no es entendida como un desarrollo alarmante (spraw), sino como una entidad socioespacial en si misma, con su propia «firma» espacial, al modo entendido por Venturi y Scott Brown ${ }^{10}$ o por Garreau ${ }^{11}$. Un fuerte referente para el estudio, en términos de la noción escalar de la centralidad como forma de entender la relación entre la parte y el todo en los sistemas espaciales, es el estudio de
Vaughan (2006) que subraya el potencial de ciertos lugares para funcionar a diferentes escalas espaciales simultáneamente (Figura 1)

El estudio define los centros como lugares multiescalares dependientes del movimiento. Este movimiento estaría originado principalmente por las propiedades configuracionales (relacionales) de cada caso con respecto al sistema espacial total: la relación entre la parte y el todo. Cómo las distintas escalas de movimiento al interior de una ciudad se superponen o se separan, sería fundamental para la generación de lugares. La definición de un centro se relaciona desde este punto de vista con la capacidad de un punto dentro de la trama urbana para crear una interfaz entre distintas escalas de movimientos.

El estudio exploró la construcción de centros suburbanos basándose en una variedad de escalas espacio-funcionales, y examinando en qué medida las distintas escalas de movimientos de los habitantes de una ciudad son generadas e integradas por el sistema espacial. Diversos estudios desarrollados bajo el marco de SS apuntan a que el sentido

\footnotetext{
9 High Street se refiere a la calle comercial (Main Street en EE.UU.), ya sea nombrada específicamente o donde la actividad comercial es más densa.

10 Learning from Las Vegas. MIT Press, 1972.
}

11 Edge city: Life on the new frontier. Anchor Books, 1992. 
de lugar y el sentido del todo surgen de propiedades extrínsecas del sistema, más que de características exclusivamente locales. Los espacios que tendemos a reconocer como bien constituidos coinciden con lugares que logran superponer distintas escalas y habitantes. Los instrumentos configuracionales desarrollados por SS están categorizados en locales y globales dependiendo de la escala en la que son calculados por los softwares. Radios globales examinan las relaciones espaciales del sistema total (todos los puntos con todos los puntos), mientras que radios locales examinan las relaciones de todos los puntos pero a ciertos radios de alcance. Sinergia es la relación estadística entre el rol global y local de un lugar ${ }^{12}$. Esta medida permite el estudio de las maneras en que las diferentes escalas de los sistemas espaciales se relacionan unas con otras. En este sentido, sinergia mide el potencial de interacción espacial y social y puede ser interpretado como la relación entre los habitantes locales y visitantes extraños en un área: la interfaz entre la comunidad local y la comunidad global. A través de los veinte casos estudiados se confirma la idea de que hay lugares donde la escala global y la escala local se superponen, y otros donde divergen. Estas diferencias se relacionan con la actividad socioeconómica de cada lugar y con el proceso de centralidad.

Londres ha sido descrita como una ciudad de pueblos absorbidos, a la manera en que Puente Alto fue absorbido por Santiago. Este grado de absorción puede ser medido y puede también explicar diferencias socioeconómicas y jerarquías dentro del sistema urbano. El estudio demuestra que la centralidad es un problema escalar; las diferentes escalas de movimiento que un sistema espacial permite pueden dar forma a determinados procesos sociales. Una primera taxonomía de los veinte casos es propuesta en base a las propiedades morfológicas de los centros, a través de un estudio de step-depth ${ }^{13}$ (Figura 2). Tres genotipos son identificados: casos concéntricos, lineares y expandidos (rojo, azul y verde, respectivamente en Figura 3).

Morfológicamente, la definición escalar de centralidad es una función del grado de absorción de cada centro con respecto al total del sistema espacial. Casos concéntricos están ubicados lejos del centro de Londres,

Figura 3: Patrones entre localización y morfología.

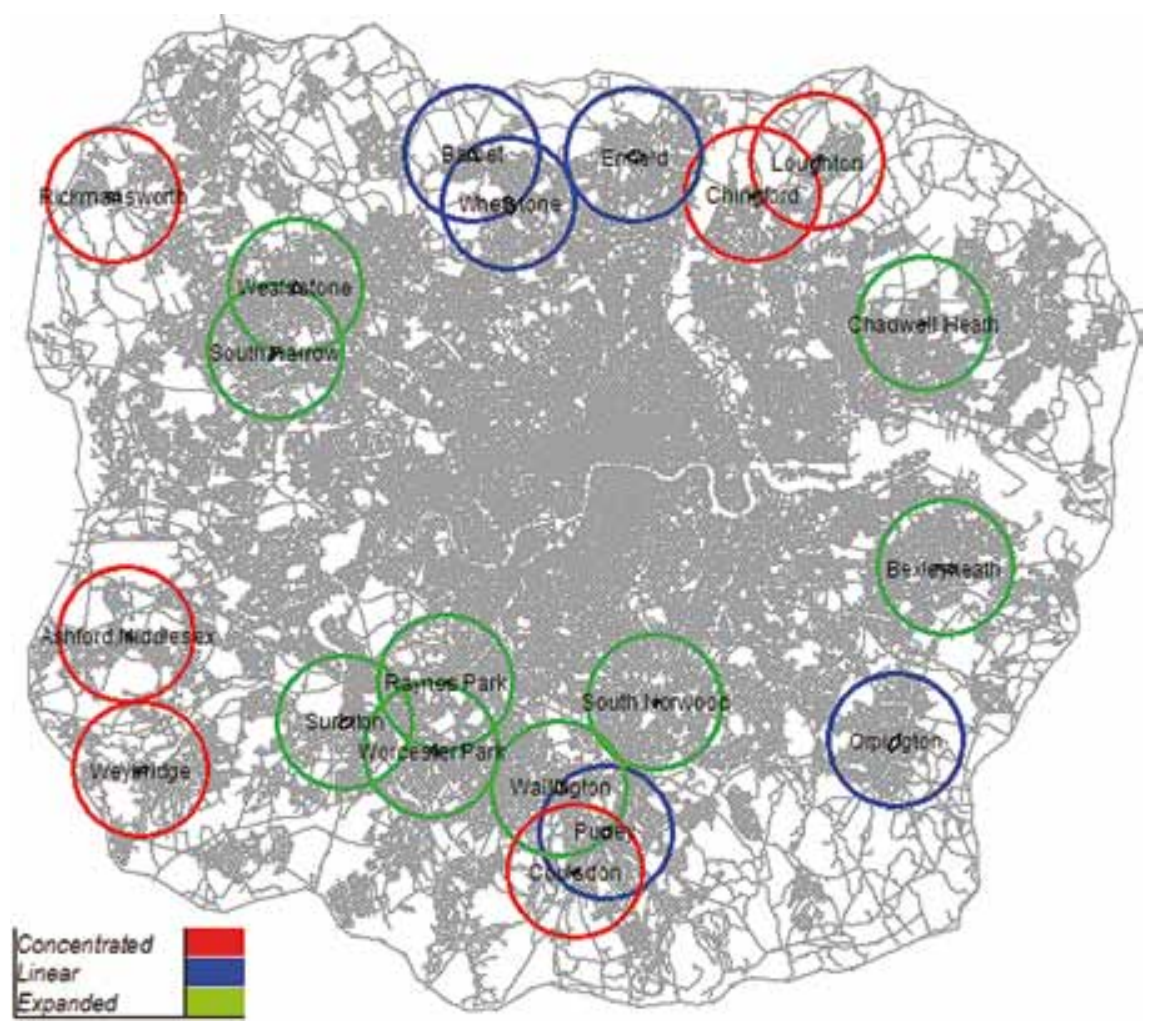

entre grandes vacíos en la trama urbana producto de los campos agrícolas y reservas de agua los cuales producen interrupciones en la continuidad de la grilla. La trama urbana del perímetro representa un estado o etapa previa en el proceso de formación de suburbia en Londres. Los casos indicados en rojo (concéntricos) están menos absorbidos por el sistema espacial total, que los indicados en azul (lineares) o en verde (expandidos) (Figura 4).

Los casos lineares están ubicados en una franja intermedia entre urbanidad y suburbanidad, entre alta y baja densidad, mientras que los casos expandidos están ubicados en tramas urbanas más continuas que se asemejan a lo que comúnmente se entiende por urbano. La Figura 5 muestra un estudio de continuidad de la trama urbana, con vacíos en negro y la trama en blanco, que ilustra claramente estas diferencias. El tamaño y número de vacíos decrece desde los casos concéntricos a los expandidos, como evidencia de un continuo entre «ruralidad», «suburbanidad» y «urbanidad». Esta taxonomía fue contrastada con la actividad socioeconómica de los casos, estableciendo el siguiente modelo de emergencia de subcentralidad:

- inicialmente, el centro suburbano se desarrolla sobre una línea corta (high street), en una sección de uno de los pasos de flujo local identificados por los análisis configuracionales (Figura 7). La actividad socioeconómica (industria y comercio principalmente) es muy localizada en torno a esta línea inicial. El índice de absorción es muy bajo, con un efecto localizante que demuestra una relativa desconexión con respecto al sistema espacial total (Figura $6)$.

12 Hillier, B. «Cities as Networks». Wolfram Science Conference in Washington, 2006.

13 Step-depth es una medida configuracional calculada en Depthmap. En un sistema espacial ciertos nodos son tomados como origen (en esta caso la High Street de cada caso). El software identifica los nodos a un paso de lejanía desde el origen y les asigna el valor 1 y un color. Si se encuentra a dos pasos de lejanía, le asigna el valor 2 y otro color, y así sucesivamente. 


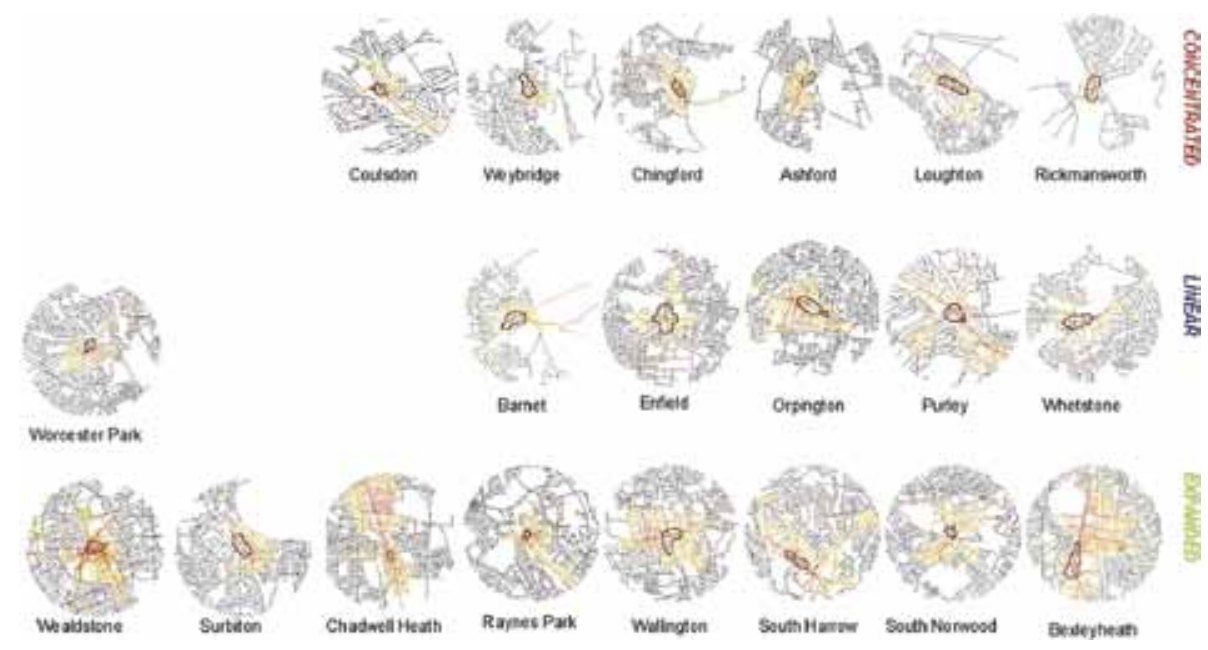

Figura 5
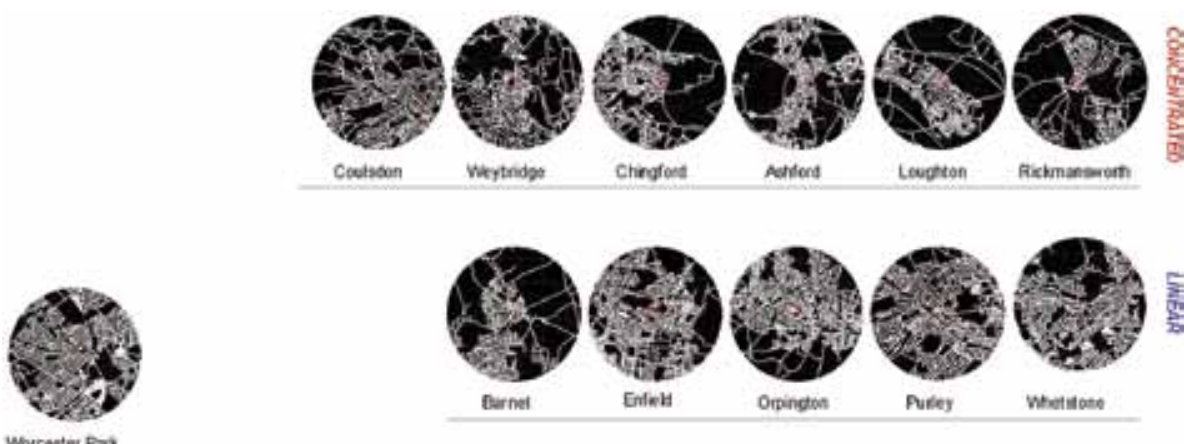

chingones

Aations

Louptor

Aikmminson

Barnet
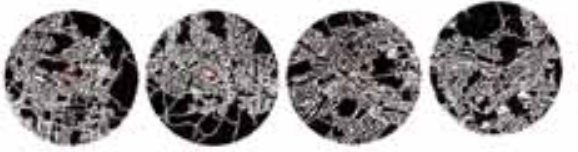

EMle:

Pustey Whetilitate
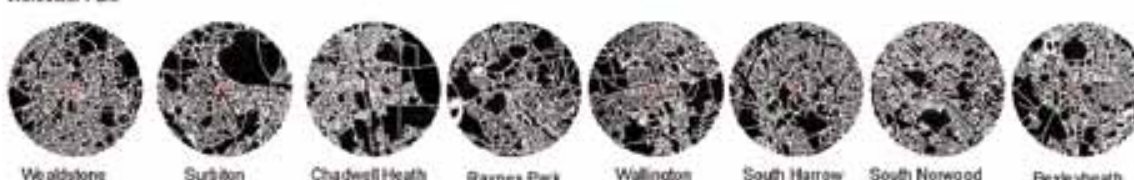

Chadentiteda

Rapnespor.

Wilingow
- A medida que el asentamiento crece, se desencadena un proceso de intensificación de la trama urbana y una diversidad de usos emergen en torno a una morfología linear, la cual es parte de viajes a mayor escala dentro de la ciudad (Figura 8). El centro está más absorbido por el principal centro urbano y ahora es parte de la red de conexiones a nivel global. El índice de absorción es mayor, y menos local (Figura $6)$

- Con mayor crecimiento, la absorción del centro por el principal centro urbano es casi completo. La industria es empujada por el comercio a una mayor distancia de la high street. A medida que el centro suburbano comienza a ser absorbido la high street ya no es parte de un paso global como en la etapa linear, sino más bien interaccesibilidad es lo que da forma a la centralidad (Figura 6).

Una segunda taxonomía es propuesta a partir del potencial de movimiento, basada en el análisis de las propiedades configuracionales de cada centro con respecto al total del sistema espacial: Local-global para aquellos casos en que la significancia espacial es mayor local que globalmente. Global-local para aquellos casos en que la significancia espacial es mayor global que localmente. Balanceados cuando existe un equilibrio entre movimientos globales y locales. Los genotipos propuestos a partir del potencial de movimiento también guardan relación estadística con procesos de origen social. Los casos con mayor significancia espacial local, tiene mayores porcentajes de suelo destinado a comercio y oficinas. Los casos con mayor significancia espacial global, tienen mayores porcentajes de suelo destinado a industria y manufactura. Los casos balanceados en que las escalas global y local se encuentran superpuestas, coinciden con una mayor diversidad de actividades, lo que se ha considerado como una señalización de emergencia de centralidad y de construcción de lugar. Esto sugiere que una estructura configuracionalmente balanceada implica a su vez formas sociales más balanceadas: la existencia de una interfaz socioespacial (Tabla 1 ).

La centralidad suburbana entonces no depende sólo de propiedades morfológicas distintivas, sino también de cómo lo local se relaciona con escalas más globales de movimiento al interior de la ciudad. Esto sugiere una noción escalar de la centralidad, asociada al grado de absorción en términos espacio-funcionales, donde en un extremo el centro histórico es completamente indistinguible dentro del sistema global y en el otro extremo el centro aún está completamente segregado de éste. La relación entre la parte y el todo en las ciudades ha sido largamente debatida por autores como Lynch $^{14}$, Mumford ${ }^{15}$ y Jacobs ${ }^{16}$, pero aún es un desafío cómo crear lugares y centralidades reconocibles. Las ideas brevemente expuestas en este artículo exploran la relación parte-todo en una ciudad policéntrica, demostrando que existen muchas maneras de ser central en una ciudad, y que la centralidad suburbana no es una condición, sino un proceso con diferentes etapas y grados en términos espacio-funcionales. Los tres genotipos morfológicos identificados (concéntricos, lineales y expandidos) y los tres genotipos extraídos del potencial de movimiento (global-local, local-global y balanceados) actúan como descripciones de estas etapas. El estudio confirma que no es la suma de partes lo que constituye un sistema espacial total, sino que es del total que surgen partes bien o mal constituidas. Las conclusiones además sugieren que mecanismos espaciales y a-espaciales son necesarios en la construcción de lugares.

14 Lynch, K. A theory of good city form. Cambridge, MA: MIT Press, 1981.

15 Mumford, L. The city in history. New Jersey: Harcourt, Brace \& World, 1961

16 Jacobs, J. The death and life of great american cities. Londres: Pimlico, 1962. 

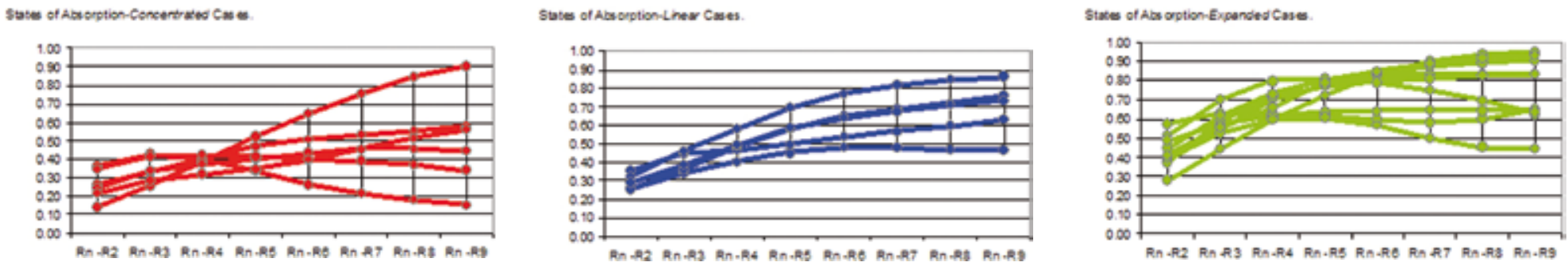

Figura 6
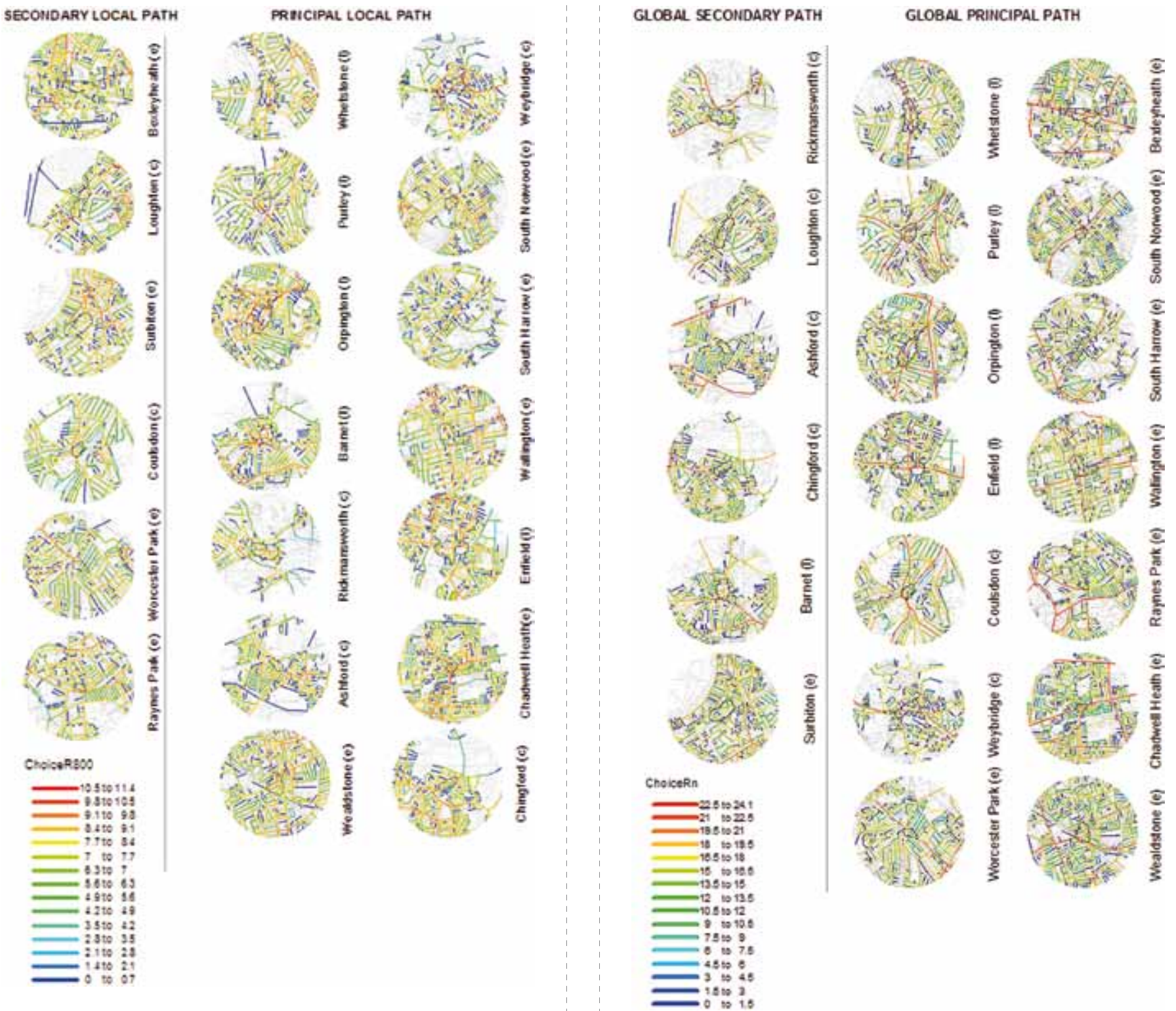

Figura 7

Figura 8 
Tabla 1: Tres genotipos de potencial de movimiento: Local-Global, Global-Local y Balanceados.

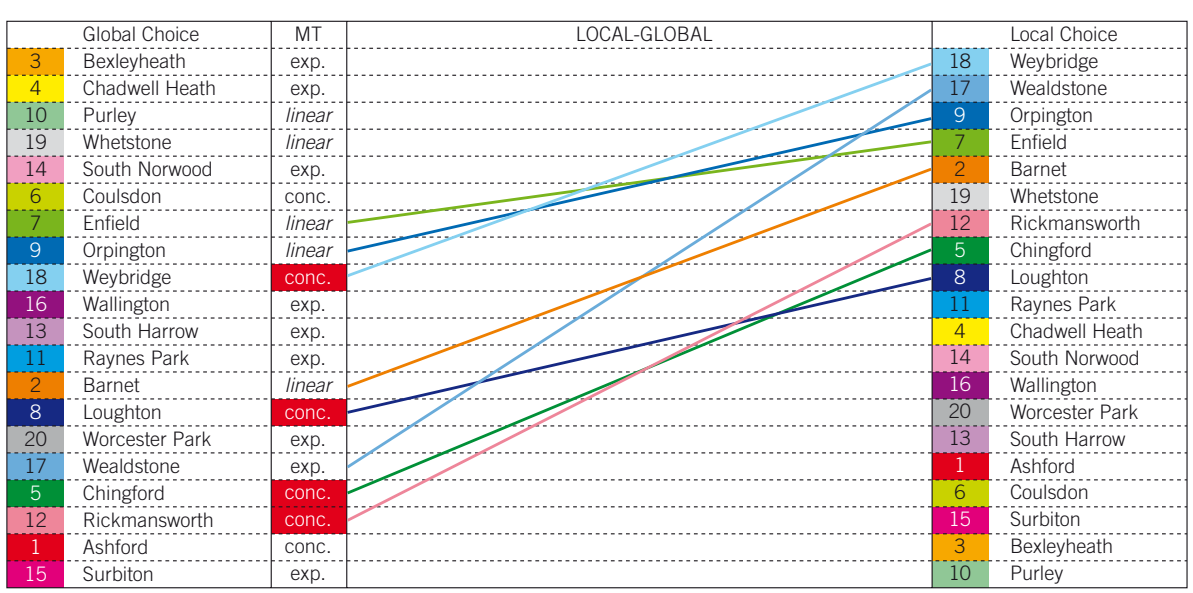

Bajo estas circunstancias, el concepto de centralidad urbana debiese ser revisado para la suburbanidad; la teoría de centralidad suburbana debería asumir la forma de una pregunta escalar.

Las nuevas posibilidades tecnológicas implican un paradigma diferente dentro del proceso diseño desde dos puntos de vista. Primero, permiten entender la relación espacio-sociedad bidireccionalmente, y segundo, diferencian el campo disciplinar entre los conceptos de orden y estructura, descritos por Hanson ${ }^{17}$. Los arquitectos históricamente hemos tendido a diseñar bajo conceptos de orden espacia (top-down) con ejemplos cúlmines en Brasilia y Chandigarh. Las herramientas y software desarrollados por SS apuntan al concepto de estructura (bottom-up) en el que ese orden visual aparente es reemplazado por la noción de estructura. Mientras el orden es inmediatamente perceptible, la estructura requiere ser develada a través de técnicas discursivas que informen el proceso de diseño. La habilidad escalar del espacio para superponer o separar diferentes escalas de movimiento y diferentes tipos de habitantes, es de relevancia no sólo en la macroescala del territorio, sino también en el diseño más acotado de espacios públicos y edificios.

\begin{tabular}{|c|c|c|c|c|c|}
\hline & Global Choice & MT & LOCAL-GLOBAL & & Local Choice \\
\hline 3 & Bexleyheath & exp. & & 18 & Weybridge \\
\hline 4 & Chadweil Heath & exp. & & 17 & Wealdstone \\
\hline 10 & Purley & linear & & 9 & Orpington \\
\hline 19 & Whetstone & linear & & 7 & Enfield \\
\hline 14 & South Norwood & exp. & & 2 & Barnet \\
\hline 6 & Coulsdon & conc. & & 19 & Whetstone \\
\hline 7 & Enfield & linear & & 12 & Rickmansworth \\
\hline 9 & Orpington & linear & & 5 & Chingford \\
\hline 18 & Weybridge & conc. & & 8 & Loughton \\
\hline 16 & Wallington & exp. & $\cdots$ & 11 & Raynes Park \\
\hline 13 & South Harrow & exp. & & 4 & Chadwell Heath \\
\hline 11 & Raynes Park & $\exp$ & 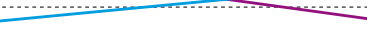 & 14 & South Norwood \\
\hline 2 & Barnet & linear & & 16 & Wallington \\
\hline 8 & Loughton & conc. & & 20 & Worcester Park \\
\hline 20 & Worcester Park & exp. & & 13 & South Harrow \\
\hline 17 & Wealdstone & exp. & & 1 & Ashford \\
\hline 5 & Chingford & conc. & & 6 & Coulsdon \\
\hline 12 & Rickmansworth & conc. & & 15 & Surbiton \\
\hline 1 & Ashford & conc. & & 3 & Bexleyheath \\
\hline 15 & Surbiton & exp. & & 10 & Purley \\
\hline
\end{tabular}

17 Hanson, J. «Order and structure in urban design: The plans for the rebuilding of London after the Great Fire of 1666 from Ekistics». Special Issue on Space Syntax 1989; 56(334-335). 